\title{
Comparison of Three Browser Performances
}

\author{
Stefanus Lintang Timur Aji Pamungkas \\ Department of Information Systems, Soegijapranata Catholic University \\ lintangtimur915@gmail.com
}

\begin{abstract}
In this era of globalization and modernity, it is important to obtain information, and the role of Internet in providing information is indispensable. The existence of the World Wide Web technology makes it easy for people to surf various websites. The elements on the website are the code javascript, css, and HTML5.
\end{abstract}

Keywords-- World Wide Web, javascript, HTML5, browser, internet.

\section{INTRODUCTION}

In search of information on the Internet, people, of course, will make use the Web Browser.

When someone is accessing a website with a particular browser, he is able to identify that the browser is different from the other. There are 3 browsers most commonly used, those are, Google Chrome, Mozilla Firefox, and Internet Explorer.

In this article, I want to discuss what make the 3 browsers different in terms of their performances to access webs.

\section{LITERATURE REVIEW}

\section{$2.1 \quad$ Internet}

Computers of one countries can be connected to those of other countries via modem devices, supplied by the internet services provider or ISP.

Some internet services available today are:

a. FTP : File Transfer Protocol. It is useful for displaying data or a file to a server on the Internet. b. E-Mail as mail delivery in electronic form.

c. World Wide Web. It is used to surf on the Internet and obtain information.

\section{RESEARCH METHODOLOGY}

To gather relevant information, the writer used questionnaire method. I distributed a questionnaire containing a comparison among the the three leading web browsers, those are, Google Chrome, Mozilla Firefox, and Internet Explorer with various benchmark tests. The finding is that as many as 55\% of respondents use Google Chrome browser, then followed by Firefox. No one uses IE.

\section{RESULTS AND DISCUSION}

Web Browser

Web Browser is an application that is used to obtain and present information from the Internet, which is then stored in a computer server. Technically, a web browser is a program that uses the Hypertext Transfer Protocol to put requests from the web server via the Internet. Most browsers are able to support E-Mail and FTP (Files Transfer Protocol)

HTML 5 Test

HTML5 Test is a web application for evaluating the accuracy of web browsers in HTML5 Standardization implementation.

WEBxprt

Webxprt is a free online tool that gives the object a series of information about how well a laptop, tablet smartphone or other devices are in handling HTML and Javascript, 
such as, face detection test or photo manipulation.

Speedometer test

Speedometer test is a test to measure how responsive a browser is to access the web application.

Jet Stream test

Jet stream test is a test javascript focusing on web application complexity.

It combines various benchmarks of javascript, which include workload and web programming techniques.

Sunspider test

It is like Jetstream, but with no complexity. Sunspider itself merely tests core benchmark test of javascript language only. Compared to some browsers, different from javascript benchmark, Sunspider test covers: Real World

This test avoids testing micro test in dept, yet focuses more on solving everyday problems encountered by developers with javascript.

Balanced

This test balances language and code differences. Not math, all the strings, or all the little things about looping. And additional test in a variety of categories.

Statiscally Sound

This benchmark runs several times to determine a range of errorr. In addition, it also functions to find out whether or not statistical differences occur.

Octane 2.0

It is a modern benchmark that measures the ability of javascript engine in running a series of representative tests complexity which demands web applications. Updating Octane 2.0 itself already contains four new assets used to measure new aspects of javascript capabilities, including in the collection of garbage or compiler.

Peacekeeper

A free online benchmarking tool, the peacekeeper can quickly and easily identify which performance of browser is better or worse in your PC. Peacekeeper ability test focuses on javascript test, on how quick a code can be implemented.

OORT Online

Online application benchmark is used to measure the performance of the browser WebGL, which is used to play online games via a browser.

Extension

Extension or Add-On is a small piece of software that can be modified to add a particular function of a website such as VPN.

My questionnaires were distributed to 29 respondents. None of them uses As many as $55 \%$ of the respondents pick up Chrome owned by Google as the most excellent one.

The most favorite browser is Google, chosen by $55.2 \%$ of the respondents, followed by Firefox, $44.8 \%$ of the respondents. Internet Explorer is not put into consideration at all. Google itself is regarded superior in terms of facilities, simple graphical interface, and being user friendly.

With Email facility, Gmail, Chrome spoil its users for it can be easily accessed anytime and anywhere. There are some respondents who have no idea about Gmail owned Google, which is equipped with Chrome features. A total of 18 responses agrees that the E-Mail of Gmail enriched with facilities of the Chrome browser. There are 2 Respondents (6.9\%) who do not agree chose not agree. The remaining 9 respondents (31\%) choose "do not know."

For a test on how good the performance of the website is in running HTML5, the technology of Chrome is considered superior in achieving the benchmark of HTML5 test. Many respondents do not realize this factt. 11 respondents agree because HTML5 technology is a new HTML element which is very important in a web. And Chrome performs very well. 3 people do not agree on HTML5 test processed through Chrome. Those who answer "do not know" are as many as 15 respondents. 
For the Speedometer benchmark test: Chrome is the best in giving responses to the website to access the web apps. Many respondents who agree to it.

18 people agree that Chrome performs quick responses. As many as 10 respondents answer "do not know." One person does not agree because he thinks that the process is not that important.

By using Google Chrome, users will be pampered with amenities by Google itself. 23 people agree on this; 3 disagree, and the other 3 answer have no idea since they prefer a different browser.

For Extensibility, Chrome is more superior. There are many data bases extensions which can be modified. There are 13 respondents who agree that the extensions in chrome are easier to use than the add-on in Firefox. As many as 7 respondents do not agree for having a different opinion, that it is easier to use add-on in Firefox instead. As many as 9 respondents who do not know about browser extension

In terms of CPU usage, Chrome has a small outlay, that is, as many as $71.1 \%$, followed by IE, 91\%. Firefox is considered to be sluggish. 11 people know and agree that Chrome is lighter for aprocess in computer. 7 people disagree about this, because they argue that Chrome has more processes than the other browsers. 11 respondents do not know and dod not observe if Firefox browser is extremely slow.

With Benchmark Octane 2.0, Chrome is the best in having access to modern webs which are complex as long as they use javascript code which is also complex. 9 people know about javascript code and agree if Chrome is more excellent in terms of benchmark. As many as 16 people have no idea on what makes Chrome become the best browser. 4 people disagree and even if they do not know the significance of javascript code in an element of a website.
A total of $62.1 \%$ of respondents, agrees that chrome displays simple and minimal design, and therefore suitable for small-sized screen tablet or PC. $24.1 \%$ of respondents do not agree that the Chrome design is simple, while the displays of other browsers can be altered. $13.8 \%$ do not know this, because they do not observe the graphical interface in chrome.

For excessively installing an extension, $69 \%$ of respondents agree that Chrome is noticeably slower in the process of accessing the website or during startup. $10.3 \%$ do not agree because firefox is slower than chrome. $20.7 \%$ do not know if installing extensions in excess will make a lag in the browser.

For the question on how good is the web in handling HTML and javascript, 48.3\% of respondents do not know if Firefox is the most excellent one in WEBxprt test, while $2.9 \%$ of respondents do not agree of Firefox is the most excellent one. The latter prefers Chrome. $44.8 \%$ of the respondents agree that Firefox is better and faster in handling the elements of website.

For the question on how quick the javascript code is and how smooth its performance is in PC, $34.5 \%$ of respondents agree that Firefox is the best in the Peacekeeper test. $48.3 \%$ of respondents do not have any idea about this matter. $17.2 \%$ of respondents does not agree, and prefers Chrome instead..

There are $79.3 \%$ of respondents who agree that the availability of Firefox Add-On is higher in number compared to the other. Few other (6.9\%) pick up firefox chrome. $13.8 \%$ do not know of the existence and functionality of the firefox add-on.

There are $55.2 \%$ of respondents agree that many Adds-On do not work if the recent version of Firefox is updated, while $37.9 \%$ of respondents dos not know that firefox updating will disable adds-on. $6.9 \%$ of respondents do not agree and prefer chrome instead. 
$41.4 \%$ of respondents agree that Firefox is the browser experiencing rapid growth, and that its users keep growing. Firefox is easier to run and it has faster starting-up. However, 27.6\% of respondents do not agree because for them, the flexibility of chrome excels that of firefox. 31\% have no idea on the nature of the browser the used.

More than $51 \%$ of respondents do not know if Internet Explorer is the best in the Sunspider test (in which only the elements elements frequently encountered in common websites which are being tested). 27.6\% of respondents do not agree because they rarely use IE. $20.7 \%$ agree because they ever use IE and they find out that the elements - elements of common websites can be quickly processed with IE.

A total of $48.3 \%$ of respondents does not know that the memory usage of Internet Explorer is at best with $3384 \mathrm{MB}$ since IE itself only focuses on the elements of common websites. $17.2 \%$ of respondents do not agree that IE is the best because it takes up a lot of memory, while $34.5 \%$ agree because at the beginning they install windows using IE, and feels light.

The menus/ command bars of, Internet explorer are too many and as a result, its display looks complicated regardless of its various modifications. though it can be modified. For users, the display is too overwhelming.

The Internet Explorer users in Windows Live will be spoiled with facilities belonging to windows live itself. This is possible because windows live is integrated to IE.

The respondents agree that the great number of Adds-On installed in Firefox make the loading process with Firefox is updated, then most adds-on do not work.

\section{CONCLUSIONS}

With the distributed questionnaires, this research finds out that many respondents prefer Google Chrome as their web browser default because with its E-Mail facility integrated with Google facilities itself, chrome browser certainly spoils its users. With a simple and minimal interface, Google Chrome is a level beyond both Mozilla Firefox and Internet Explorer. For interface, IE has too many commanda/menus which make it look complicated. For benchmark test, Chrome is more superior than the other. It also excels in terms of memory usage, graphical interface, and the degree of friendly usage, and variety of features.

\section{REFERENCES}

[1]http://www.principledtechnologies.com/ben chmarkxprt/webxprt/faq

[2]http://winpoin.com/inilah-web-browserterbaik-2014-edge-vs-firefox-vs-chromevs-ie-vs-opera/

[3]http://www.academia.edu/4072821/jurnal_il miah

[4]http://searchwindevelopment.techtarget.com /definition/browser

[5]http://wokopad.com/wiki/HTML5test

[6]http://www.principledtechnologies.com/ben chmarkxprt/webxprt/faq

[7]http://www.principledtechnologies.com/ben chmarkxprt/webxprt/run-webxprt-mobile

[8]http://browserbench.org/Speedometer/

[9]http://browserbench.org/JetStream/indepth.html

[10]https://www.webkit.org/perf/sunspider/sun spider.html

[11]https://developers.google.com/octane/

[12]http://peacekeeper.futuremark.com/faq.acti on

[13]http://www.makeuseof.com/tag/peacekeep er-benchmark-performance-battery-lifedevice/

[14]http://oortonline.gl/ 